\title{
The Real-World Efficacy and Safety of Anlotinib in Advanced Non-Small Cell Lung Cancer
}

\section{Fen Wang ( $\square$ fina_wang@163.com )}

Peking University Shenzhen Hospital https://orcid.org/0000-0002-4876-710X

\section{Feng Jin}

Peking University Shenzhen Hospital

\section{Boran Cheng}

Peking University Shenzhen Hospital

\section{Yue Zhang}

Peking University Shenzhen Hospital

\section{Qing Zhou}

Guangdong Provincial People's Hospital

\section{Shubin Wang}

Peking University Shenzhen Hospital

\section{Research Article}

Keywords: anlotinib, non-small cell lung cancer, real-world, tyrosine kinase inhibitor, anti-angiogenesis

Posted Date: March 11th, 2021

DOI: https://doi.org/10.21203/rs.3.rs-282608/v1

License: (9) (i) This work is licensed under a Creative Commons Attribution 4.0 International License. Read Full License

Version of Record: A version of this preprint was published at Journal of Cancer Research and Clinical Oncology on August 6th, 2021. See the published version at https://doi.org/10.1007/s00432-021-03752-x. 


\section{Abstract}

Purpose: Anlotinib is an anti-angiogenetic multi-targeted tyrosine kinase inhibitor. This study aimed to evaluate the efficacy and safety of anlotinib in advanced non-small cell lung cancer (aNSCLC) in the real world.

Methods: Patients with aNSCLC receiving anlotinib were enrolled in two cohorts (treatment-naive and previously treated). The endpoints included progression-free survival (PFS), overall survival (OS) and anlotinib-related adverse events (ar-AEs).

Results: 203 patients accrued in the study. In the treatment-naïve cohort $(n=80)$, the PFS was 7.4 (95\% confidence interval [CI], 4.1-10.7) and OS was 10.8 ( $95 \% \mathrm{Cl}, 5.8-15.8)$ months of monotherapy group (immature survival for combination group). In previously treated cohort $(\mathrm{n}=123)$, The PFS was 8.0 months $(95 \% \mathrm{Cl}, 6.1-9.9)$ in the combination group and 4.3 months (95\% $\mathrm{Cl}, 2.1-6.6)$ in the monotherapy group (hazard ratio [HR], $0.49 ; 95 \% \mathrm{Cl}, 0.29-0.83 ; \mathrm{p}=0.007$ ), respectively. The $0 \mathrm{~S}$ was 18.5 months $(95 \% \mathrm{Cl}, 10.5$ - to 26.6$)$ in the combination group and 7.8 months $(95 \% \mathrm{Cl}, 7.1-8.4)$ in the monotherapy group (HR, $0.38 ; 95 \% \mathrm{Cl}, 0.22-0.66 ; \mathrm{p}=0.001)$, respectively. The ar-AEs of grade $\geq 3$ in the monotherapy and the combination groups were hypertension ( $9.0 \%$ and $8.7 \%$ ), fatigue ( $8.1 \%$ and $7.6 \%$ ), hand-foot syndrome ( $8.1 \%$ and $6.5 \%)$, diarrhea (5.4\% and $8.7 \%)$, proteinuria ( $5.4 \%$ and $5.4 \%)$, and mucositis oral $(6.3 \%$ and $8.7 \%)$.

Conclusion: In aNSCLC, anlotinib monotherapy has a promising efficacy in the first-line setting. It may be an option for those who are ineligible for chemotherapy; anlotinib combination therapy in a $\geq$ second-line setting showed manageable toxicities and encouraging efficacy, indicating a good application prospect.

Trial registration: This study was retrospectively registered with ISRCTN Registry (ID ISRCTN35543977) on January $26^{\text {th }}$, 2021 and Chinese Clinical Trial Register (ChiCTR2000032265) on April $4^{\text {th }}, 2020$.

\section{Introduction}

Non-small cell lung cancer (NSCLC) is the commonest type of lung cancer and comprises $83 \%$ of all lung cancers (Miller et al. 2019), with $\geq 50 \%$ of cases diagnosed at advanced stages (Siegel et al. 2019). Following the increased understanding of the molecular and immunologic profiles of lung cancer and advances in targeted therapy and immunotherapy, the treatment of advanced NSCLC (aNSCLC) has greatly advanced in the last 20 years. The 5-year overall survival (OS) of patients with aNSCLC has greatly improved with the use of these agents (Garon et al. 2019; Gettinger et al. 2018; Lin et al. 2016; Mok et al. 2009; Ramalingam et al. 2020).

Anti-angiogenesis therapy remains indispensable in the standard care for aNSCLC since it normalizes the tumor vasculature and suppresses the tumor microenvironment. The standard first-line treatment for aNSCLC without oncogenic driver mutations in China is platinum-based chemotherapy (Barlesi et al. 2014; Sandler et al. 2006; Zhou et al. 2015), or in combination with bevacizumab, if non-squamous (Fossella et al. 2003; Scagliotti et al. 2008; Schiller et al. 2002). Initial chemotherapy plus a programmed death-ligand 1 (PD-L1) inhibitor has not proven to be more beneficial than a combination with bevacizumab (Socinski et al. 2020). In patients with epidermal growth factor receptor (EGFR)-mutated aNSCLC, a combination of anti-angiogenesis therapy and EGFR-tyrosine kinase inhibitors (TKIs) has improved patient survival than EGFR-TKI alone (Nakagawa et al. 2019; Saito et al. 2019; Zhou et al. 2019b).

Anlotinib is an oral small molecular multi-targeted TKI with anti-angiogenic (by inhibiting vascular endothelial growth factor receptor 1-3 and fibroblast growth factor receptor 1-4) and anti-tumor proliferation properties (by inhibiting platelet-derived growth factor receptor $\alpha$ and $\beta$, RET, and stem cell factor receptor) (Lin et al. 2018; Sun et al. 2016; Xie et al. 2018). It has been approved by the National Medical Products Administration of China for $\geq$ third-line treatment of aNSCLC (Han et al. 2018) and advanced small cell lung cancer (Cheng et al. 2018).

Notably, substantial distinct exists between the patients in the clinical trials and in the real world, particularly those with poor conditions (e.g., the elderly, patients with a performance status [PS] $\geq 2$, brain metastases, and comorbidities) (Nabhan et al. 
2019). However, anlotinib may be preferred in clinical practice for patients with aNSCLC who are not eligible or unwilling to receive standard care. Several preclinical and clinical trials have confirmed the synergy between anti-angiogenesis therapy and chemotherapy, targeted therapy and immunotherapy, providing a rationale for a combination therapy strategy with these regimens (Alshangiti et al. 2018). Furthermore, oral anlotinib therapy during the coronavirus disease (COVID-19) pandemic has several advantages such as reducing the number of in-person visits or invasive procedures. Therefore, we investigated the efficacy and safety of anlotinib when used alone or with other antineoplastic agents in patients with aNSCLC in a realworld setting. This is the first of such studies.

\section{Materials And Methods}

\section{Study Design and Patients}

This was a retrospective observational cohort study conducted at Peking University Shenzhen Hospital, a university-affiliated tertiary hospital located in Guangdong, China.

Adult (age $\geq 18$ years) patients treated with anlotinib or anlotinib-containing regimens between 1 June 2018 and 30 September 2020 were identified through electronic medical order system (EMS). Patients were screened if they had pathologically confirmed stage IIIB to IV or recurrent NSCLC, measurable disease as evaluated based on Response Evaluation Criteria in Solid Tumours (RECIST, version 1.1) (Eisenhauer et al. 2009), adequate organs function and a performance status of 0 to 3. Eligible patients were enrolled in treatment-naïve cohort and previously treated cohort. Each cohort was divided into two groups, monotherapy and combination therapy. Exclusion criteria included, incomplete treatment information, local treatment including interventional therapy and radiotherapy for the target lesions during anlotinib treatment, and malignancies other than lung cancer within 5 years (except those treated with curative intent and had negligible risk of death or metastases, according to the discretion of primary investigator).

\section{Procedure}

Anlotinib was administered orally once daily at an initial dose from $10 \mathrm{mg}$ to $12 \mathrm{mg}$ on day 1 to day 14 of a 21 -day cycle. The initial dose and combined regimen were decided by the physicians. Anlotinib was continued until tumour progression, death, unacceptable toxicity, and could continue beyond progression for as long as clinical benefit was observed, as judged by the physicians. Dose reductions or suspension were allowed if patients had a $\geq$ grade 3 anlotinib-related adverse events (ar-AEs). If ar-AEs resolved or reverted to $\leq$ grade 2 within 2 weeks, anlotinib was re-administrated at the same dose or a lower dose. Where ar-AEs persisted after 2-weeks interruption, anlotinib was discontinued permanently.

Tumour responses were assessed based on RECIST 1.1 every 6 to 12 weeks or significant progression occurred or necessary. Treatment beyond imaging progression and the efficacy evaluation according to the physicians' assessment in clinical practice was also allowed. Ar-AEs were categorised and graded according to the National Cancer Institute-Common Terminology Criteria for Adverse Events (NCI-CTCAE, version 4.03).

The EMS was used to collect baseline characteristics, laboratory data, AEs and outcomes. Baseline characteristics included gender, age, smoking status, Eastern Cooperative Oncology Group (ECOG) PS, pathological type, date of diagnosis of advanced disease, disease stage, metastases site, comorbidity and complication, medical histology, presence of oncogenic diver mutations (EGFR, ROS-1, RET, ALK, KRAS, BRAF, c-MET, HER2), prior and subsequent systematic treatment, and best tumor response. The biochemical parameter values and blood cell counts were collected at baseline and during anlotinib treatment.

\section{Outcomes}

The primary endpoint was progression-free survival (PFS) and secondary endpoints included objective response rate (ORR), disease control rate (DCR), overall survival (OS), and toxicity. The exploratory endpoint was potential biomarker analysis for 
anlotinib first-line monotherapy. The type of combined agents was the major stratification factor used to analyze the efficacy of later-line anlotinib-combined therapy.

The PFS was defined as the time from the first anlotinib administration to the documented disease progression or death due to any cause. The ORR and DCR was defined as the percentage of patients with at least one confirmed response and response plus stable disease before any evidence of progression, respectively. OS was defined as the time from the first anlotinib administration to death from any cause or last follow-up. Ar-AEs, including events that led to dose reductions, treatment discontinuation, or death, were collected. Lymphocyte to monocyte ratio (LMR), neutrophil to lymphocyte ratio $(\mathrm{NLR})$, platelet to lymphocyte ratio (PLR), and prognostic nutritional index (PNI, as calculated as $10 \times$ albumin level $(\mathrm{g} / \mathrm{dl})+$ $0.005 \times$ total lymphocyte count (per mm3) (Wang et al. 2018) were calculated based on the baseline laboratory data.

\section{Statistical Analysis}

Patients' baseline characteristics were reported with descriptive statistics as proportions for categorical variables and medians (range) for continuous variables. Pearson's Chi-square test or Fisher's exact test were used to compare categorical variables and tumour responses between two groups. The median follow-up period was computed based on the reverse Kaplan-Meier method. The median PFS, OS and 95\% confidence interval (CI) were estimated using the Kaplan-Meier method, with differences between groups being evaluated using the Log-rank test. Cox proportional hazards regression was used for the univariable and multivariable analysis of PFS and OS and to calculate the hazard ratios (HR) with 95\% Cls. All statistical analyses were performed using the statistical package for the social sciences (SPSS) software version 23 (SPSS Inc., Chicago, IL, USA).

\section{Ethical Approval and Informed Consent}

The study was approved by the China Ethics Committee of Registering Clinical Trials (No. ChiECRCT20200083) and performed in accordance with Good Clinical Practice and the provisions of the Declaration of Helsinki. Written informed consent was waived given the nature of the study.

\section{Results}

\section{Patients and treatment}

From June 1, 2018, to September 30, 2020, 226 patients were screened from the EMS. A total of 203 patients were enrolled: 80 in the treatment-naïve cohort and 123 in the previously treated cohort (Figure 1). The median follow-up duration was 11.0 months (range, 7.1-14.8) and 10.0 months (range, 8.8-11.8), respectively.

Details of the patients' characteristics are shown in Table 1. Patients aged $\geq 75$ years (22 [27.5\%] and 15 [12.2\%], respectively), had PS $\geq 2$ (28 [35.0\%] and 40 [32.5\%], respectively), and had central nervous system metastases (7 [8.8\%] and 23 [18.7\%], respectively) were also enrolled, although patients with these characteristics are usually under-represented in clinical trials. There were more male patients $(84.6 \%$ vs. $60.7 \%, p=0.017)$ in the treatment-naïve monotherapy group than in the combination group, and more patients with PS $\geq 2(44.1 \%$ vs. $21.9 \%, p=0.009)$ in the previously treated monotherapy group.

\section{PFS and OS}

In the treatment-naïve cohort, 22 events of disease progression or death occurred in the monotherapy group and 5 in the combination group, respectively; 35 and 1 deaths occurred in the monotherapy and combination groups, respectively. Given the limited events of the combination group in this cohort, we analyzed the survival of the monotherapy group only. The median PFS was 7.4 months (95\% confidence interval [CI], 4.1-10.7). The median OS was 10.8 months $(95 \% \mathrm{Cl}, 5.8-15.8)$ in the treatment-naïve monotherapy group (Figure 2). 
In the previously treated cohort, 63 events of disease progression or death occurred. The median PFS was 8.0 months (95\% $\mathrm{Cl}, 6.1-9.9)$ in the combination group and 4.3 months $(95 \% \mathrm{Cl}, 2.1-6.6)$ in the monotherapy group (hazard ratio [HR], $0.49 ; 95 \%$ $\mathrm{Cl}, 0.29-0.83 ; \mathrm{P}=0.007$ ), respectively (Figure $3 \mathrm{~A}$ ). The HR for PFS was less than 1.00 across almost all subgroups except for the patients with ECOG PS $\geq 2$ and harbouring diver mutations (Figure 3B). However, the upper boundaries of the $95 \% \mathrm{Cl}$ crossed 1.00 for multiple subgroups. The interaction test showed the treatment efficacy varied significantly only across the subgroups of histology types (non-squamous vs. squamous, $p=0.045$ ) and driver mutations (yes vs. no, $p=0.034$ ).

With 59 deaths, the median OS was 18.5 months $(95 \% \mathrm{Cl}, 10.5-26.6)$ in the combination group and 7.8 months $(95 \% \mathrm{Cl}$, 7.18.4 ) in the monotherapy group ( $\mathrm{HR}, 0.38 ; 95 \% \mathrm{Cl}, 0.22-0.66 ; \mathrm{P}=0.001)$, respectively (Figure $4 \mathrm{~A})$. Consistent with PFS, the OS benefit of the combination was observed in all subgroups (Figure 4B), yet with the upper boundaries of the $95 \%$ Cls crossing 1.00 in multiple subgroups. No statistical differences in treatment efficacy comparing combination with monotherapy were observed among subgroups in the interaction test. The PFS and OS did not differ among patients who received different anlotinib-combined agents (median PFS, ICls vs. TKIs vs. chemotherapy: 18.2 vs. 7.6 vs. 8.0 months, $p=0.483$; median OS, ICls vs. TKls vs. chemotherapy: 18.5 vs. not reach [NR] vs. 19.6 months, $p=0.348$ ) (Supplemental, Figure 5A and B).

In the multivariate analysis, the combination $(p=0.044)$ and the prior anti-angiogenesis treatment $(p=0.013)$ were statistically associated with the PFS; whereas only the combination $(\mathrm{p}=0.010)$ was statistically associated with the OS (Supplemental, Table 2).

\section{Tumour response}

In the treatment-naïve cohort, no patient in the monotherapy group and one patient in the combination group achieved CR; 7 and 11 achieved PR in the two groups, respectively. The ORR was significantly higher in the combination group than that in the monotherapy group ( $42.9 \%$ vs. $13.5 \%, p=0.004)$; while the DCR was only numerically higher without a statistical difference (82.1\% vs. $73.1 \%, p=0.197)$ (Supplemental, Table 3).

In the previously treated cohort, more patients responded to anlotinib combination therapy, with 1 (1.6\%) CR and 18 (28.1\%) $\mathrm{PR}$, respectively. There were significant differences in both ORR and DCR between the combination and monotherapy groups (ORR, $29.7 \%$ vs. $6.8 \%, p=0.002$; DCR, $81.4 \%$ vs. $59.3 \%$, $p=0.025$ ) (Supplemental, Table 3 ).

\section{Exploratory analysis}

In the treatment-naïve monotherapy group, the potential predictive and prognostic value of patients' characteristics and several laboratory parameters, including NLR, LMR, PLR, and PNI, were exploratory analyzed. No clinical characteristics were significantly associated with PFS or OS in the univariate analysis (Supplemental, Table 4). The median baseline values of these parameters (NLR, 4.1; LMR, 2.2; PLR, 188.3; PNI, 39.9) were used as cut-off values to distinguish between patients with low and high values. Notably, high baseline LMR was significantly associated with improved PFS (HR, 0.37; 95\% Cl, 0.14-0.99; $p=0.048)$, while high PNI was associated with longer OS (HR, 0.33; 95\% Cl, 0.14-0.76; $p=0.009)$ (Supplemental Table 4). NLR and PLR were not significantly associated with PFS and OS. Only high PNI was statistically associated with improved OS (HR, 0.34; 95\% Cl, 0.13-0.90; $\mathrm{p}=0.030$ ) in the multivariate analysis using the previous factors (data not shown).

\section{Safety Analysis}

The toxicity of monotherapy $(n=111)$ and combination therapy $(n=92)$ was analyzed in all the patients. The toxicities are summarized in Table 5. The most prevalent anlotinib-related side effects in the monotherapy group were hypertension (51.3\%), fatigue (47.7\%), anorexia (39.6\%), hand-foot syndrome (34.2\%), cough (32.4\%), diarrhea (30.6\%), hypothyroidism (29.7\%), proteinuria (29.7\%), and oral mucositis (25.2\%); whereas those of anlotinib-combined therapy included fatigue $(71.7 \%)$, anorexia $(62.0 \%)$, rash (51.1\%), hypertension (48.9\%), oral mucositis (45.7\%), diarrhea (43.5\%), hand-foot syndrome (39.1\%), and hypothyroidism (39.1\%). Most of these toxicities were grade $1-2$. At least $5 \%$ of the grade $\geq 3$ adverse events in the monotherapy and combination groups were hypertension ( $9.0 \%$ and $8.7 \%)$, fatigue ( $8.1 \%$ and $7.6 \%)$, hand-foot syndrome 
(8.1\% and $6.5 \%)$, diarrhea (5.4\% and $8.7 \%$ ), proteinuria (5.4\% and $5.4 \%)$, and oral mucositis (6.3\% and $8.7 \%$, respectively). The only grade $\geq 3$ adverse event that was more frequent in the combination group was anorexia $(8.7 \%$ and $0.9 \%)$.

Dose reductions were required for 5 (4.5\%) patients in the monotherapy group, including 4 (3.6\%) cases of first-level reduction and $1(0.9 \%)$ case of second-level reduction. Dose reductions occurred in $5(5.4 \%)$ patients in the combination group (all onelevel reductions). The suspension rates were $3.6 \%(4 / 111)$ and $6.5 \%(6 / 99)$ in the two groups, respectively.

\section{Discussion}

In this study, the survival of treatment-naïve patients with aNSCLC who received anlotinib monotherapy was promising. Also, patients with aNSCLC who received a combination of anlotinib and other anti-tumor agents had significantly improved survivals than those treated with anlotinib alone, administered as $\otimes$ second-line therapy). A combination of vascular endothelial growth factor (VEGF) monoclonal antibodies and chemotherapy improved the PFS and OS in first-line (bevacizumab combination) (Patel et al. 2013; Reck et al. 2009; Sandler et al. 2006; Zhou et al. 2015) and later-line (ramucirumab combination) (Garon et al. 2014; Shiono et al. 2019) settings of aNSCLC, respectively. In multiple phase III trials, the combination of VEGF monoclonal antibodies with erlotinib as first-line treatment significantly improved the PFS in patients with EGFR-mutant aNSCLC than erlotinib alone (Nakagawa et al. 2019; Saito et al. 2019; Zhou et al. 2019a). However, multi-target TKIs that mainly block the VEGF signaling pathway have exhibited mixed clinical activity in aNSCLC. Compared to placebo, these agents (e.g., vandetanib, pazopanib, sunitinib, and sorafenib) failed to improve survival in aNSCLC (Natale et al. 2011; Paz-Ares et al. 2015; Scagliotti et al. 2012a; Weiss et al. 2014). In combination with chemotherapy, unlike anlotinib, none of them had a superior efficacy to chemotherapy alone in aNSCLC (de Boer et al. 2011; Goss et al. 2010; Hanna et al. 2016; Herbst et al. 2010; Laurie et al. 2014; Lee et al. 2012; Paz-Ares et al. 2012; Reck et al. 2014; Scagliotti et al. 2010; Scagliotti et al. 2013; Scagliotti et al. 2012b). In the ALTER0303 trial, anlotinib greatly prolonged the OS and PFS than placebo. Anlotinib has a wide range of specific targets, including c-FMS, Aurora B, and discoidin domain receptor 1 (a group of newly identified kinase targets involved in tumor progression) (Sun et al. 2016), which might explain its positive results in aNSCLC. Likewise, the inhibitory action of these targets is responsible for anlotinib's anti-tumor and antiangiogenic properties, which could explain its encouraging efficacy in the treatment-naïve monotherapy group in our study. In previous clinical trials of first-line treatment in aNSCLC, bevacizumab plus chemotherapy significantly prolonged the PFS from 4.5 months to 6-6.7 months, and OS from 10.3 months to 12.3-13.4 months (Patel et al. 2013; Reck et al. 2009; Sandler et al. 2006). In our study, first-line anlotinib monotherapy demonstrated a comparable PFS (> 7 months) and slightly worse OS (approximate 11 months) than that of bevacizumab plus chemotherapy. These results may be due to the unique mechanism of anlotinib or the study population. Several patients in the treatment-naïve monotherapy group were wild-type mutations and squamous cell carcinomas, who lacks effective first-line targeted therapy. However, no clinical characteristics, including histology and diver mutations, was significantly associated with PFS or OS in this group. This is consistent with the ALTER0303 trial wherein anlotinib was effective for EGFR-mutant and wild-type patients (Han et al. 2018), with a significant improvement in the PFS, but not OS of patients with squamous cell carcinomas. The fibroblast growth factor (FGF) signaling pathway plays a key role in squamous cell lung cancer by promoting tumor cell proliferation and cancer angiogenesis through several mechanisms (Helsten et al. 2015; Procopio et al. 2015; Tiseo et al. 2015). As against FGF receptor, anlotinib the efficacy of anlotinib in this population can be explained. Of note, anlotinib was administered to a population with a heavy tumor burden and adverse prognosis in our study (30-40\% of patients were $\geq 75$-years old, had ECOG PS $\geq 2$ or multiple metastases). Thus, the therapeutic effect of anlotinib may be undervalued because of the recipients' poor conditions and shorter life expectancy. Moreover, the limited rescue treatment in this population might have contributed to the shorter OS than that of standard bevacizumab-containing chemotherapy. Anlotinib monotherapy has less survival benefits as first-line treatment than immunochemotherapy (Gadgeel et al. 2020; Gandhi et al. 2018), especially in terms of OS. Nevertheless, according to our findings, anlotinib may still be an appropriate choice for patients with advanced age, poor PS, or reluctance to receive chemotherapy. 
Anti-angiogenesis therapy can normalize abnormal vascularization in tumors, improve delivery of anti-tumor agents (Alshangiti et al. 2018), modify the immunosuppressive microenvironment, and crosslink with the EGFR signaling pathway (Tian et al. 2020); and thus play a synergistic antitumor role when combined with other treatments including chemotherapy, EGFR/TKIs, and ICls. Additionally, the good tolerance and non-overlapping toxicity spectrum of anlotinib makes it possible to be combined. In the previously treated cohort in our study, anlotinib combination therapy improved survival and tumor response to monotherapy. The ECOG PS of the enrolled patients was different between the two groups, which may be a cause of bias. However, it did not show significant association with the survival. Prior anti-angiogenesis therapy was also different between the two groups; and it was significantly associated with PFS, but not OS in the multivariate analysis. However, the PFS benefit of the combination was observed in the subgroups with or without previous antiangiogenic therapy, although the upper limit of the $95 \% \mathrm{Cl}$ exceeded 1 . These findings are consistent with those in earlier studies, which showed that previous antiangiogenic therapy had no influence on PFS and OS of $\geq$ third-line anlotinib treatment in aNSCLC (Han et al. 2018; Shao et al. 2019; Zhang et al. 2020).

The patients with squamous cell carcinoma and without driver mutations had a greater chance of improved PFS following combination therapy, with a $p$ value for interaction $\leq 0.05$. In the ALTER0303 trial, anlotinib had no additional therapeutic advantage in patients with squamous cell carcinoma or without driver mutations; therefore, we assume that the drugs in the combination may explain this difference. A considerable proportion of patients in the two subgroups (non-squamous vs. squamous, $36 \%$ vs. $0 \%$; driver mutations yes vs. no, $63 \%$ vs. $2.7 \%$ ) received anlotinib combined with original EGFR TKIs as rescue therapy, immediately after the treatment failure with front-line EGFR-TKIs, which might only lead to modest survival benefits. In contrast, survival benefits were significant in patients with squamous cell carcinoma and wild-type mutations, most of who switched from later-line therapy to a combination with chemotherapy or immunotherapy. In the survival analysis with combined agents as the stratification factor, patients who received the EGFR-TKI combination had the shortest PFS, but the difference was not statistically significant. Several studies have compared erlotinib plus bevacizumab with erlotinib alone as first-line treatment in EGFR-mutant aNSCLC; PFS was improved, but not OS (Kato et al. 2018; Maemondo et al. 2020; Saito et al. 2019; Zhou et al. 2019a). Consistently, the first-line combination of anlotinib with EGFR TKIs has been reported to have an extremely high ORR and DCR in patients with EGFR-mutant aNSCLC (Huang et al. 2020a). Although there is no evidence that adding anti-angiogenesis therapy can improve survival after first-line EGFR-TKI resistance, this combination strategy has still been attempted in patients with slow disease progression in clinical practice. A study on the efficacy of anlotinib combined with first-generation (1G) EGFR-TKls as second-line therapy in patients with secondary resistance to prior $1 \mathrm{G}$ EGFR TKIs and non-T790M mutations in aNSCLC is ongoing (NCT03766490).

The efficacy of anlotinib combination therapy in aNSCLC was promising in previous studies, with an ORR of $60 \%-92.6 \%$ in the first-line setting and 26\%-37.5\% in the second-line setting (B et al. 2019; Huang et al. 2020a; Wu et al. 2020). Due to the short follow-up time, the PFS was about 5 months in the patients who received anlotinib plus chemotherapy as $\geq$ second-line treatment in only two studies (Wu et al. 2020; Zhang et al. 2020). This was consistent with our study wherein anlotinib combination therapy had a better tumor response than monotherapy in both the treatment-naïve and previously treated patients; this effect was successfully translated into survival benefits in previously treated patients (the survival was immature in treatment-naïve patients).

Generally, the safety profile of anlotinib combination therapy was comparable to that of monotherapy in terms of the frequency of $\geq$ grade 3 treatment-related adverse events and dose modification. The side effects in our study were in accordance with those in prior studies (Han et al. 2018; Huang et al. 2020a; Huang et al. 2020b; Wu et al. 2020; Zhang et al. 2020). There were no new safety concerns or anlotinib-related deaths.

The identification of predictive and prognostic factors of anti-angiogenic treatment is challenging. In our exploratory analysis, the PFS and OS were improved significantly in patients with higher baseline LMRs in the anlotinib monotherapy group, suggesting that LMR might be a predictor of the efficacy of anlotinib in this setting. LMR is a prognostic factor in lung cancer (Chen et al. 2015; Go et al. 2014; Hu et al. 2014; Song et al. 2016). Decreased LMR was shown to have significantly negative correlation with PFS and OS in bevacizumab treatment in aNSCLC (Li et al. 2019). The reason for this is unknown. We 
hypothesized that fewer circulating monocytes may reflect the limited formation or presence of tumor-associated macrophages (Clear et al. 2010; Lin et al. 2011); the latter has a positive relationship with extracellular matrix remodeling, angiogenesis, and lymphangiogenesis (Clear et al. 2010; Lin et al. 2006). Conversely, lymphocytopenia is an important component of low LMR; it induces fewer tumor-infiltrating lymphocytes for tumor cell eradication, which is associated with worse efficacy and survival in multiple malignancies (Chen et al. 2012); and it is also correlated with vascular invasion in NSCLC (Kobayashi et al. 2012). Therefore, a high LMR may reflect less angiogenesis, lymphangiogenesis, and vascular invasion, which may facilitate anlotinib treatment. Consistent with previous studies (Hong et al. 2015; Li et al. 2018), PNI was an independent prognostic factor in patients with aNSCLC who received anlotinib first-line monotherapy in our study. A high $\mathrm{PNI}$ is associated with an adequate anticancer immunological reaction, and functional and nutritional status of the host, which can enhance the tolerance and compliance to the treatment in patients with cancer (Deme and Telekes 2018; Fruchtenicht et al. 2015; Paccagnella et al. 2011). Although this is an exploratory and post hoc analysis, these markers are readily available and inexpensive in clinical practice; they could help to predict the efficacy of anlotinib and estimate the prognosis once our results are validated in future studies.

Our study has few limitations beyond the retrospective design and consequent selection bias. First, as a real-world study, the non-diverse Chinese population and small sample size might have affected the objectivity of the results. Second, the monotherapy and combination groups were clinically heterogeneous; however, the multivariate Cox regression analysis was used to adjust for confounding factors. Third, we were unable to obtain the survival of the first-line combination group due to the short follow-up duration.

Nevertheless, our study has several advantages. First, it provides a promising alternative chemotherapy-free strategy for the first-line treatment of patients with aNSCLC, especially for patients who are senile, have poor physical conditions, and unwilling or unable to receive chemotherapy. Second, in this era when first-line immunotherapy \pm chemotherapy is the standard care for patients without driver mutations, it provides additional evidence for the application of second-line antiangiogenesis combination therapy. Third, we further explored the potential predictive and prognostic factors of anlotinib monotherapy for aNSCLC, which can serve as baseline data for further studies on biomarkers of antiangiogenic therapy. Finally, the convenience and feasibility of anlotinib, especially during the COVID-19 pandemic, makes our findings generalizable.

\section{Conclusions}

Anlotinib monotherapy has a promising efficacy in the first-line setting. It may be an option for patients with aNSCLC who are ineligible for chemotherapy in the real world. Anlotinib plus other anti-tumor regimens in $a \geq$ second-line setting showed manageable toxicities and encouraging efficacy, indicating a good application prospect in aNSCLC. Our conclusion would benefit from the addition of information on the scope for further research on the topic.

\section{Declarations}

\section{Funding}

This study was funded by the Wu Jieping Medical Foundation (320.6750.19088-10), Beijing Bethune Charitable Foundation (BQE-TY-SSPC(2)-S-01), and Shenzhen Sanming Project (SZSM201612041).

\section{Conflicts of interest/Competing interests}

Dr. Qing Zhou reports speaker fees from AstraZeneca and Roche. The other authors indicate no conflicts of interest.

\section{Availability of data and material}

The datasets analyzed during the current study can be obtained from the corresponding author on reasonable requirements. 


\section{Authors' contributions}

Fen Wang: Conceptualization, Methodology, Investigation, Writing - review \& editing. Feng Jin: Investigation, Writing - review \& editing. Boran Cheng: Investigation, Writing - review \& editing. Yue Zhang: Methodology, Investigation. Qing Zhou: Conceptualization, Methodology, Investigation, Writing - review \& editing. Shubin Wang: Conceptualization, Methodology, Investigation, Writing - review \& editing.

\section{Ethics approval}

The study was approved by the China Ethics Committee of Registering Clinical Trials (No. ChiECRCT20200083) and performed in accordance with Good Clinical Practice and the provisions of the Declaration of Helsinki.

\section{Consent to participate}

Written informed consent was waived given the nature of the study.

\section{Consent for publication}

Informed consent was obtained from the authors.

\section{Acknowledgments}

We would like to thank Editage (www.editage.cn) for English language editing.

\section{References}

1. Alshangiti A, Chandhoke G, Ellis PM (2018) Antiangiogenic therapies in non-small-cell lung cancer Curr Oncol 25:S45S58 doi:10.3747/co.25.3747

2. B H, T C, R Z, al. e (2019) Efficacy and safety of sintilimab with anlotinib as first-line therapy for advanced non-small cell lung cancer (NSCLC). . Paper presented at the 2019 World Conference on Lung Cancer, Barcelona, Spain,

3. Barlesi F et al. (2014) Maintenance bevacizumab-pemetrexed after first-line cisplatin-pemetrexed-bevacizumab for advanced nonsquamous nonsmall-cell lung cancer: updated survival analysis of the AVAPERL (MO22089) randomized phase III trial Ann Oncol 25:1044-1052 doi:10.1093/annonc/mdu098

4. Chen KJ, Zhou L, Xie HY, Ahmed TE, Feng XW, Zheng SS (2012) Intratumoral regulatory T cells alone or in combination with cytotoxic T cells predict prognosis of hepatocellular carcinoma after resection Med Oncol 29:1817-1826 doi:10.1007/s12032-011-0006-x

5. Chen YM et al. (2015) Baseline and Trend of Lymphocyte-to-Monocyte Ratio as Prognostic Factors in Epidermal Growth Factor Receptor Mutant Non-Small Cell Lung Cancer Patients Treated with First-Line Epidermal Growth Factor Receptor Tyrosine Kinase Inhibitors PLoS One 10:e0136252 doi:10.1371/journal.pone.0136252

6. Cheng Y et al. (2018) Anlotinib as Third-Line or Further-Line Treatment in Relapsed SCLC: A Multicentre, Randomized, Double-Blind Phase 2 Trial Journal of Thoracic Oncology 13:S351-S352

7. Clear AJ et al. (2010) Increased angiogenic sprouting in poor prognosis FL is associated with elevated numbers of CD163+ macrophages within the immediate sprouting microenvironment Blood 115:5053-5056 doi:10.1182/blood-2009$11-253260$

8. de Boer RH et al. (2011) Vandetanib plus pemetrexed for the second-line treatment of advanced non-small-cell lung cancer: a randomized, double-blind phase III trial J Clin Oncol 29:1067-1074 doi:10.1200/JC0.2010.29.5717

9. Deme D, Telekes A (2018) [Prognostic importance of albumin in oncology] Orv Hetil 159:96-106 doi:10.1556/650.2018.30885 
10. Eisenhauer EA et al. (2009) New response evaluation criteria in solid tumours: revised RECIST guideline (version 1.1) Eur J Cancer 45:228-247 doi:10.1016/j.ejca.2008.10.026

11. Fossella F et al. (2003) Randomized, multinational, phase III study of docetaxel plus platinum combinations versus vinorelbine plus cisplatin for advanced non-small-cell lung cancer: the TAX 326 study group J Clin Oncol 21:3016-3024 doi:10.1200/JC0.2003.12.046

12. Fruchtenicht AV, Poziomyck AK, Kabke GB, Loss SH, Antoniazzi JL, Steemburgo T, Moreira LF (2015) Nutritional risk assessment in critically ill cancer patients: systematic review Rev Bras Ter Intensiva 27:274-283 doi:10.5935/0103507X.20150032

13. Gadgeel S et al. (2020) Updated Analysis From KEYNOTE-189: Pembrolizumab or Placebo Plus Pemetrexed and Platinum for Previously Untreated Metastatic Nonsquamous Non-Small-Cell Lung Cancer J Clin Oncol 38:1505-1517 doi:10.1200/JC0.19.03136

14. Gandhi L et al. (2018) Pembrolizumab plus Chemotherapy in Metastatic Non-Small-Cell Lung Cancer N Engl J Med 378:2078-2092 doi:10.1056/NEJMoa1801005

15. Garon EB et al. (2014) Ramucirumab plus docetaxel versus placebo plus docetaxel for second-line treatment of stage IV non-small-cell lung cancer after disease progression on platinum-based therapy (REVEL): a multicentre, double-blind, randomised phase 3 trial Lancet 384:665-673 doi:10.1016/S0140-6736(14)60845-X

16. Garon EB et al. (2019) Five-Year Overall Survival for Patients With Advanced NonSmall-Cell Lung Cancer Treated With Pembrolizumab: Results From the Phase I KEYNOTE-001 Study J Clin Oncol 37:2518-2527 doi:10.1200/JC0.19.00934

17. Gettinger S et al. (2018) Five-Year Follow-Up of Nivolumab in Previously Treated Advanced Non-Small-Cell Lung Cancer: Results From the CA209-003 Study J Clin Oncol 36:1675-1684 doi:10.1200/JC0.2017.77.0412

18. Go SI et al. (2014) Prognostic significance of the lymphocyte-to-monocyte ratio in patients with small cell lung cancer Med Oncol 31:323 doi:10.1007/s12032-014-0323-y

19. Goss GD et al. (2010) Randomized, double-blind trial of carboplatin and paclitaxel with either daily oral cediranib or placebo in advanced non-small-cell lung cancer: NCIC clinical trials group BR24 study J Clin Oncol 28:49-55 doi:10.1200/JC0.2009.22.9427

20. Han B et al. (2018) Effect of Anlotinib as a Third-Line or Further Treatment on Overall Survival of Patients With Advanced Non-Small Cell Lung Cancer: The ALTER 0303 Phase 3 Randomized Clinical Trial JAMA Oncol 4:1569-1575 doi:10.1001/jamaoncol.2018.3039

21. Hanna NH et al. (2016) Nintedanib plus pemetrexed versus placebo plus pemetrexed in patients with relapsed or refractory, advanced non-small cell lung cancer (LUME-Lung 2): A randomized, double-blind, phase III trial Lung Cancer 102:65-73 doi:10.1016/j.lungcan.2016.10.011

22. Helsten T, Schwaederle M, Kurzrock R (2015) Fibroblast growth factor receptor signaling in hereditary and neoplastic disease: biologic and clinical implications Cancer Metastasis Rev 34:479-496 doi:10.1007/s10555-015-9579-8

23. Herbst RS et al. (2010) Vandetanib plus docetaxel versus docetaxel as second-line treatment for patients with advanced non-small-cell lung cancer (ZODIAC): a double-blind, randomised, phase 3 trial Lancet Oncol 11:619-626 doi:10.1016/S1470-2045(10)70132-7

24. Hong $\mathrm{S}$ et al. (2015) The prognostic nutritional index (PNI) predicts overall survival of small-cell lung cancer patients Tumour Biol 36:3389-3397 doi:10.1007/s13277-014-2973-y

25. Hu P, Shen H, Wang G, Zhang P, Liu Q, Du J (2014) Prognostic significance of systemic inflammation-based lymphocytemonocyte ratio in patients with lung cancer: based on a large cohort study PLoS One 9:e108062 doi:10.1371/journal.pone.0108062

26. Huang D, Zhong D, Zhang C, Zhang Y, Shang Y, Wang L (2020a) Study of anlotinib combined with icotinib as the first-line treatment in non-small cell lung cancer (NSCLC) patients harboring activating EGFR mutations (ALTER-L004) Journal of Clinical Oncology 38:9573-9573 doi:10.1200/JCO.2020.38.15_suppl.9573

Page $10 / 22$ 
27. Huang D, Zhong D, Zhang C, Zhang Y, Shang Y, Wang L (2020b) Study of anlotinib combined with icotinib as the first-line treatment in non-small cell lung cancer (NSCLC) patients harboring activating EGFR mutations (ALTER-L004). Paper presented at the 2020 American Society of Clinical Oncology, Chicago, USA.,

28. Kato T et al. (2018) Erlotinib Plus Bevacizumab Phase Il Study in Patients with Advanced Non-small-Cell Lung Cancer (J025567): Updated Safety Results Drug Saf 41:229-237 doi:10.1007/s40264-017-0596-0

29. Kobayashi N, Usui S, Kikuchi S, Goto Y, Sakai M, Onizuka M, Sato Y (2012) Preoperative lymphocyte count is an independent prognostic factor in node-negative non-small cell lung cancer Lung Cancer 75:223-227 doi:10.1016/j.lungcan.2011.06.009

30. Laurie SA et al. (2014) Randomised, double-blind trial of carboplatin and paclitaxel with daily oral cediranib or placebo in patients with advanced non-small cell lung cancer: NCIC Clinical Trials Group study BR29 Eur J Cancer 50:706-712 doi:10.1016/j.ejca.2013.11.032

31. Lee JS et al. (2012) Vandetanib Versus placebo in patients with advanced non-small-cell lung cancer after prior therapy with an epidermal growth factor receptor tyrosine kinase inhibitor: a randomized, double-blind phase III trial (ZEPHYR) J Clin Oncol 30:1114-1121 doi:10.1200/JC0.2011.36.1709

32. Li B et al. (2019) The Kinetic Changes of Systemic Inflammatory Factors during Bevacizumab Treatment and Its Prognostic Role in Advanced Non-small Cell Lung Cancer Patients J Cancer 10:5082-5089 doi:10.7150/jca.30478

33. Li D, Yuan X, Liu J, Li C, Li W (2018) Prognostic value of prognostic nutritional index in lung cancer: a meta-analysis J Thorac Dis 10:5298-5307 doi:10.21037/jtd.2018.08.51

34. Lin B, Song X, Yang D, Bai D, Yao Y, Lu N (2018) Anlotinib inhibits angiogenesis via suppressing the activation of VEGFR2, PDGFRbeta and FGFR1 Gene 654:77-86 doi:10.1016/j.gene.2018.02.026

35. Lin EY et al. (2006) Macrophages regulate the angiogenic switch in a mouse model of breast cancer Cancer Res 66:11238-11246 doi:10.1158/0008-5472.CAN-06-1278

36. Lin JJ, Cardarella S, Lydon CA, Dahlberg SE, Jackman DM, Janne PA, Johnson BE (2016) Five-Year Survival in EGFRMutant Metastatic Lung Adenocarcinoma Treated with EGFR-TKIs J Thorac Oncol 11:556-565 doi:10.1016/j.jtho.2015.12.103

37. Lin JY, Li XY, Tadashi N, Dong P (2011) Clinical significance of tumor-associated macrophage infiltration in supraglottic laryngeal carcinoma Chin J Cancer 30:280-286 doi:10.5732/cjc.010.10336

38. Maemondo M et al. (2020) NEJ026: Final overall survival analysis of bevacizumab plus erlotinib treatment for NSCLC patients harboring activating EGFR-mutations Journal of Clinical Oncology 38:9506-9506 doi:10.1200/JC0.2020.38.15_suppl.9506

39. Miller KD et al. (2019) Cancer treatment and survivorship statistics, 2019 CA Cancer J Clin 69:363-385 doi:10.3322/caac. 21565

40. Mok TS et al. (2009) Gefitinib or carboplatin-paclitaxel in pulmonary adenocarcinoma N Engl J Med 361:947-957 doi:10.1056/NEJMoa0810699

41. Nabhan C, Klink A, Prasad V (2019) Real-world Evidence-What Does It Really Mean? JAMA Oncol 5:781-783 doi:10.1001/jamaoncol.2019.0450

42. Nakagawa K et al. (2019) Ramucirumab plus erlotinib in patients with untreated, EGFR-mutated, advanced non-small-cell lung cancer (RELAY): a randomised, double-blind, placebo-controlled, phase 3 trial Lancet Oncol 20:1655-1669 doi:10.1016/S1470-2045(19)30634-5

43. Natale RB et al. (2011) Phase III trial of vandetanib compared with erlotinib in patients with previously treated advanced non-small-cell lung cancer J Clin Oncol 29:1059-1066 doi:10.1200/JC0.2010.28.5981

44. Paccagnella A, Morassutti I, Rosti G (2011) Nutritional intervention for improving treatment tolerance in cancer patients Curr Opin Oncol 23:322-330 doi:10.1097/CCO.0b013e3283479c66 
45. Patel JD et al. (2013) PointBreak: a randomized phase III study of pemetrexed plus carboplatin and bevacizumab followed by maintenance pemetrexed and bevacizumab versus paclitaxel plus carboplatin and bevacizumab followed by maintenance bevacizumab in patients with stage IIIB or IV nonsquamous non-small-cell lung cancer J Clin Oncol 31:4349-4357 doi:10.1200/JC0.2012.47.9626

46. Paz-Ares L et al. (2015) Monotherapy Administration of Sorafenib in Patients With Non-Small Cell Lung Cancer (MISSION) Trial: A Phase III, Multicenter, Placebo-Controlled Trial of Sorafenib in Patients with Relapsed or Refractory Predominantly Nonsquamous Non-Small-Cell Lung Cancer after 2 or 3 Previous Treatment Regimens J Thorac Oncol 10:1745-1753 doi:10.1097/JT0.0000000000000693

47. Paz-Ares LG et al. (2012) Phase III, randomized, double-blind, placebo-controlled trial of gemcitabine/cisplatin alone or with sorafenib for the first-line treatment of advanced, nonsquamous non-small-cell lung cancer $\mathrm{J}$ Clin Oncol 30:30843092 doi:10.1200/JC0.2011.39.7646

48. Procopio MG et al. (2015) Combined CSL and p53 downregulation promotes cancer-associated fibroblast activation Nat Cell Biol 17:1193-1204 doi:10.1038/ncb3228

49. Ramalingam SS et al. (2020) Overall Survival with Osimertinib in Untreated, EGFR-Mutated Advanced NSCLC N Engl J Med 382:41-50 doi:10.1056/NEJMoa1913662

50. Reck M et al. (2014) Docetaxel plus nintedanib versus docetaxel plus placebo in patients with previously treated nonsmall-cell lung cancer (LUME-Lung 1): a phase 3, double-blind, randomised controlled trial Lancet Oncol 15:143-155 doi:10.1016/S1470-2045(13)70586-2

51. Reck M et al. (2009) Phase III trial of cisplatin plus gemcitabine with either placebo or bevacizumab as first-line therapy for nonsquamous non-small-cell lung cancer: AVAil J Clin Oncol 27:1227-1234 doi:10.1200/JC0.2007.14.5466

52. Saito H et al. (2019) Erlotinib plus bevacizumab versus erlotinib alone in patients with EGFR-positive advanced nonsquamous non-small-cell lung cancer (NEJ026): interim analysis of an open-label, randomised, multicentre, phase 3 trial Lancet Oncol 20:625-635 doi:10.1016/S1470-2045(19)30035-X

53. Sandler A et al. (2006) Paclitaxel-carboplatin alone or with bevacizumab for non-small-cell lung cancer N Engl J Med 355:2542-2550 doi:10.1056/NEJMoa061884

54. Scagliotti G et al. (2010) Phase III study of carboplatin and paclitaxel alone or with sorafenib in advanced non-small-cell lung cancer J Clin Oncol 28:1835-1842 doi:10.1200/JC0.2009.26.1321

55. Scagliotti GV et al. (2013) An open-label, multicenter, randomized, phase II study of pazopanib in combination with pemetrexed in first-line treatment of patients with advanced-stage non-small-cell lung cancer J Thorac Oncol 8:15291537 doi:10.1097/JT0.0000000000000005

56. Scagliotti GV et al. (2012a) Sunitinib plus erlotinib versus placebo plus erlotinib in patients with previously treated advanced non-small-cell lung cancer: a phase III trial J Clin Oncol 30:2070-2078 doi:10.1200/JC0.2011.39.2993

57. Scagliotti GV et al. (2008) Phase III study comparing cisplatin plus gemcitabine with cisplatin plus pemetrexed in chemotherapy-naive patients with advanced-stage non-small-cell lung cancer J Clin Oncol 26:3543-3551 doi:10.1200/JC0.2007.15.0375

58. Scagliotti GV et al. (2012b) International, randomized, placebo-controlled, double-blind phase III study of motesanib plus carboplatin/paclitaxel in patients with advanced nonsquamous non-small-cell lung cancer: MONET1 J Clin Oncol 30:2829-2836 doi:10.1200/JC0.2011.41.4987

59. Schiller JH et al. (2002) Comparison of four chemotherapy regimens for advanced non-small-cell lung cancer $\mathrm{N}$ Engl $\mathrm{J}$ Med 346:92-98 doi:10.1056/NEJMoa011954

60. Shao L, Wang W, Song Z, Zhang Y (2019) The efficacy and safety of anlotinib treatment for advanced lung cancer Onco Targets Ther 12:6549-6554 doi:10.2147/OTT.S205674

61. Shiono A et al. (2019) Improved efficacy of ramucirumab plus docetaxel after nivolumab failure in previously treated non-small cell lung cancer patients Thorac Cancer 10:775-781 doi:10.1111/1759-7714.12998

62. Siegel RL, Miller KD, Jemal A (2019) Cancer statistics, 2019 CA Cancer J Clin 69:7-34 doi:10.3322/caac.21551

Page 12/22 
63. Socinski MA et al. (2020) Impower150 Final Analysis: Efficacy of Atezolizumab and Chemotherapy Bevacizumab in First-line Metastatic Non-squamous Non-small Cell Lung Cancer Across Key Subgroups. Paper presented at the AACR, The USA,

64. Song YJ, Wang LX, Hong YQ, Lu ZH, Tong Q, Fang XZ, Tan J (2016) Lymphocyte to monocyte ratio is associated with response to first-line platinum-based chemotherapy and prognosis of early-stage non-small cell lung cancer patients Tumour Biol 37:5285-5293 doi:10.1007/s13277-015-4397-8

65. Sun Y et al. (2016) Safety, pharmacokinetics, and antitumor properties of anlotinib, an oral multi-target tyrosine kinase inhibitor, in patients with advanced refractory solid tumors J Hematol Oncol 9:105 doi:10.1186/s13045-016-0332-8

66. Tian W, Cao C, Shu L, Wu F (2020) Anti-Angiogenic Therapy in the Treatment of Non-Small Cell Lung Cancer Onco Targets Ther 13:12113-12129 doi:10.2147/OTT.S276150

67. Tiseo M et al. (2015) FGFR as potential target in the treatment of squamous non small cell lung cancer Cancer Treat Rev 41:527-539 doi:10.1016/j.ctrv.2015.04.011

68. Wang Z, Wang Y, Zhang X, Zhang T (2018) Pretreatment prognostic nutritional index as a prognostic factor in lung cancer: Review and meta-analysis Clin Chim Acta 486:303-310 doi:10.1016/j.cca.2018.08.030

69. Weiss JM, Villaruz LC, Socinski MA, Ivanova A, Grilley-Olson J, Dhruva N, Stinchcombe TE (2014) A single-arm phase II trial of pazopanib in patients with advanced non-small cell lung cancer with non-squamous histology with disease progression on bevacizumab containing therapy Lung Cancer 86:288-290 doi:10.1016/j.lungcan.2014.08.011

70. Wu L et al. (2020) A randomized phase II multi-institutional trial of anlotinib plus docetaxel versus docetaxel in EGFRwild-type non-small cell lung cancer (NSCLC) patients after progression on first-line platinum-base chemotherapy: ALTER-L018 Journal of Clinical Oncology 38:e21703-e21703 doi:10.1200/JC0.2020.38.15_suppl.e21703

71. Xie C, Wan X, Quan H, Zheng M, Fu L, Li Y, Lou L (2018) Preclinical characterization of anlotinib, a highly potent and selective vascular endothelial growth factor receptor-2 inhibitor Cancer Sci 109:1207-1219 doi:10.1111/cas.13536

72. Zhang K, Ma X, Gao H, Wang H, Qin H, Yang S, Liu X (2020) Efficacy and Safety of Anlotinib in Advanced Non-Small Cell Lung Cancer: A Real-World Study Cancer Manag Res 12:3409-3417 doi:10.2147/CMAR.S246000

73. Zhou C et al. (2015) BEYOND: A Randomized, Double-Blind, Placebo-Controlled, Multicenter, Phase III Study of First-Line Carboplatin/Paclitaxel Plus Bevacizumab or Placebo in Chinese Patients With Advanced or Recurrent Nonsquamous Non-Small-Cell Lung Cancer J Clin Oncol 33:2197-2204 doi:10.1200/JC0.2014.59.4424

74. Zhou Q et al. (2019a) 14800 - CTONG 1509: Phase III study of bevacizumab with or without erlotinib in untreated Chinese patients with advanced EGFR-mutated NSCLC Annals of Oncology 30:v603 doi:10.1093/annonc/mdz260.002

75. Zhou Q et al. (2019b) CTONG 1509: Phase 3 study of bevacizumab with or without erlotinib in untreated Chinese patients with advanced EGFR-mutated NSCLC. Paper presented at the ESMO,

\section{Tables}


Table 1

Patients baseline characteristics in two cohorts.

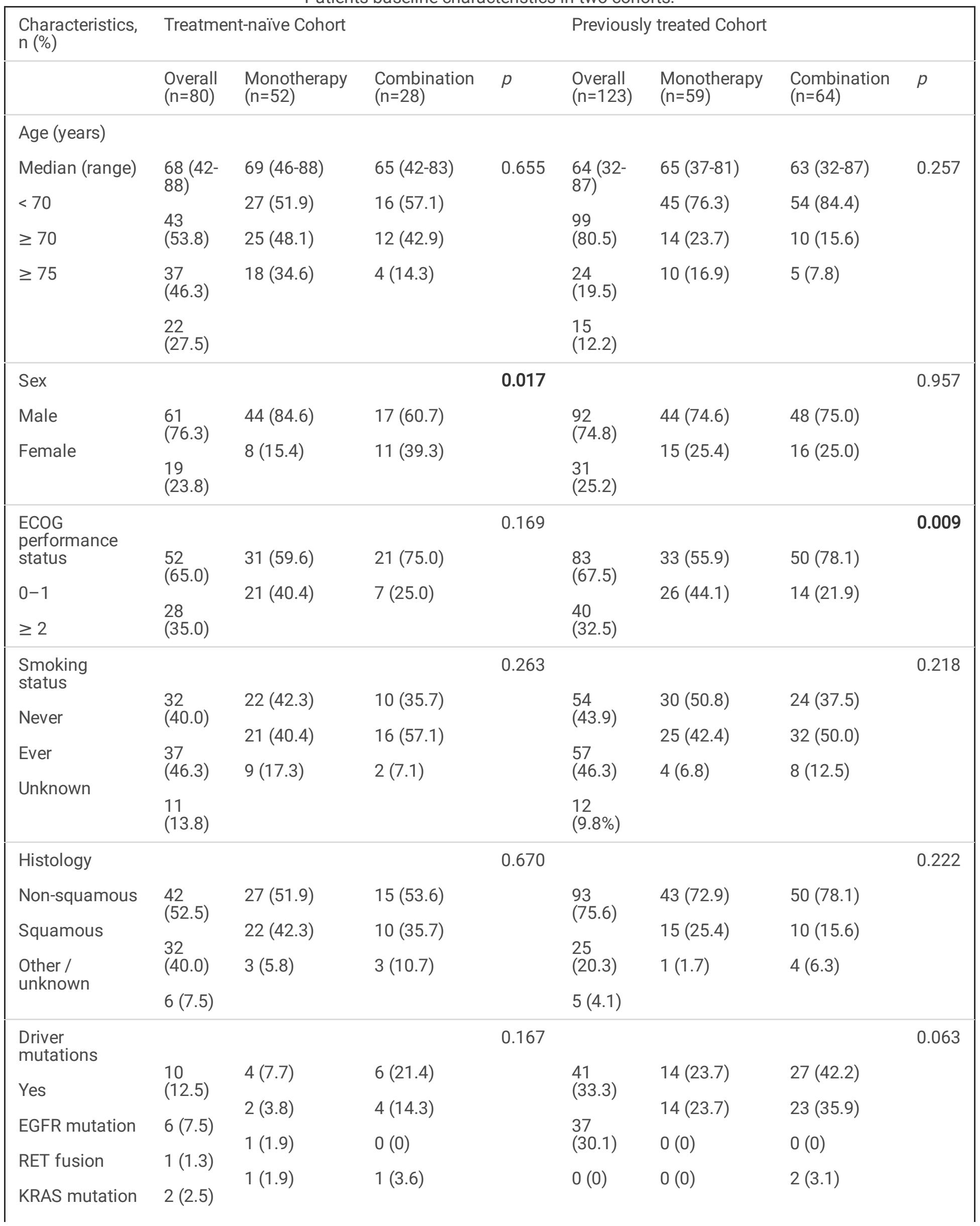

Page $14 / 22$ 


\begin{tabular}{|c|c|c|c|c|c|c|c|c|}
\hline BRAF mutation & $1(1.3)$ & $0(0)$ & $1(3.6)$ & & $2(1.6)$ & $0(0)$ & $0(0)$ & \\
\hline HER2 mutation & $0(0)$ & $0(0)$ & $0(0)$ & & $0(0)$ & $0(0)$ & $2(3.1)$ & \\
\hline MET & $0(0)$ & $0(0)$ & $1(3.6)$ & & $2(1.6)$ & $0(0)$ & $0(0)$ & \\
\hline amplifıcatıon a & 69 & $47(90.4)$ & $22(78.6)$ & & $0(0)$ & $44(74.6)$ & $37(57.8)$ & \\
\hline & (86.3) & $1(1.3)$ & $0(0)$ & & 81 & $1(1.7)$ & $0(0)$ & \\
\hline Unknown & $1(1.3)$ & & & & (65.9) & & & \\
\hline & & & & & $1(0.8)$ & & & \\
\hline Stage & & & & 0.310 & & & & 0.646 \\
\hline IIIB / IIIC & 8 & $7(13.5)$ & $1(3.6)$ & & $12(9.8)$ & $5(8.5)$ & $7(10.9)$ & \\
\hline IV / Recurrent & $(10.0)$ & 45 (86.5) & 27 (96.4) & & 111 & 54 (91.5) & 57 (89.1) & \\
\hline & $\begin{array}{l}72 \\
(90.0)\end{array}$ & & & & (90.2) & & & \\
\hline Number of & & & & 0.813 & & & & 0.236 \\
\hline $\begin{array}{l}\text { sites } \\
\text { situc }\end{array}$ & 39 & $24(46.2)$ & $15(53.6)$ & & 39 & $23(39.0)$ & $16(25.0)$ & \\
\hline$<3$ & & $22(42.3)$ & 10 (35.7) & & (01.1) & $31(52.5)$ & $40(62.5)$ & \\
\hline$\geq 3$ & $(40.0)$ & $6(11.5)$ & 3 (10.7) & & $\begin{array}{l}71 \\
(57.7)\end{array}$ & $5(8.5)$ & $8(12.5)$ & \\
\hline $\begin{array}{l}\text { Other / } \\
\text { unknown }\end{array}$ & $\begin{array}{l}9 \\
(11.2)\end{array}$ & & & & $\begin{array}{l}13 \\
(10.6)\end{array}$ & & & \\
\hline CNS & & & & 0.967 & & & & 0.347 \\
\hline & $7(8.8)$ & $4(7.7)$ & $3(10.7)$ & & 23 & 9 (15.3) & $14(21.9)$ & \\
\hline res & 73 & 48 (92.3) & 25 (89.3) & & $(18.1)$ & 50 (84.7) & 50 (78.1) & \\
\hline No & (91.3) & & & & $\begin{array}{l}100 \\
(81.3)\end{array}$ & & & \\
\hline Anlotinib initial & & & & 1.000 & & & & 0.709 \\
\hline . & 75 & 49 (94.2) & 26 (92.9) & & 116 & 55 (93.2) & 61 (95.3) & \\
\hline & & $3(5.8)$ & $2(7.1)$ & & & $4(6.8)$ & $3(4.7)$ & \\
\hline $10 \mathrm{mg}$ & $5(6.3)$ & & & & $7(5.7)$ & & & \\
\hline Combined & - & - & & & - & - & & \\
\hline $1 \mathrm{Cla}$ & & & 18 (64.3) & & & & $27(42.2)$ & \\
\hline$T K$ & & & $5(17.9)$ & & & & $18(28.1)$ & \\
\hline 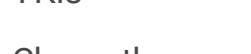 & & & 5 (17.9) & & & & 19 (29.7) & \\
\hline & & & & & & & & \\
\hline $\begin{array}{l}\text { Number of } \\
\text { nnlotinih lines }\end{array}$ & - & - & - & & & & & 0.465 \\
\hline 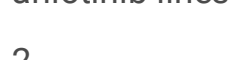 & & & & & 48 & $25(42.4)$ & $23(35.9)$ & \\
\hline 2 & & & & & & 34 (57.6) & 41 (64.1) & \\
\hline$\geq 3$ & & & & & $\begin{array}{l}75 \\
(61.0)\end{array}$ & & & \\
\hline $\begin{array}{l}\text { Prior anti- } \\
\text { angiogenesis }\end{array}$ & - & - & - & & & & & 0.065 \\
\hline & & & & & 50 & $29(49.2)$ & $21(32.8)$ & \\
\hline Yes & & & & & $(40.7)$ & $30(50.8)$ & $43(67.2)$ & \\
\hline No & & & & & $\begin{array}{l}73 \\
(59.3)\end{array}$ & & & \\
\hline
\end{tabular}

Page 15/22 
a One patient harboured KRAS mutation and MET amplification simultaneously.

ECOG, Eastern Cooperative Oncology Group; EGFR, epidermal growth factor receptor; ICI, immune checkpoint inhibitor; $T K I$, tyrosine kinase inhibitor; CNS, central nervous system; -, not applicable 
Table 5

Summary of toxicities in monotherapy and combination patients.

\begin{tabular}{|c|c|c|c|c|}
\hline \multirow[t]{2}{*}{ Toxicity } & \multicolumn{2}{|c|}{ Monotherapy ( $n=111)$, no. (\%) } & \multicolumn{2}{|c|}{ Combination $(n=92)$, no. $(\%)$} \\
\hline & All grades & Grade $\geq 3$ & All grades & Grade $\geq 3$ \\
\hline \multicolumn{5}{|l|}{ Symptoms } \\
\hline Hypertension & $57(51.3)$ & $10(9.0)$ & 45 (48.9) & $8(8.7)$ \\
\hline Fatigue & $53(47.7)$ & $9(8.1)$ & $66(71.7)$ & $7(7.6)$ \\
\hline Anorexia & $44(39.6)$ & $1(0.9)$ & $57(62.0)$ & $8(8.7)$ \\
\hline Hand-foot syndrome & $38(34.2)$ & $9(8.1)$ & $36(39.1)$ & $6(6.5)$ \\
\hline Cough & $36(32.4)$ & $3(2.7)$ & $27(29.3)$ & $0(0)$ \\
\hline Diarrhea & $34(30.6)$ & $6(5.4)$ & $40(43.5)$ & $8(8.7)$ \\
\hline Hypothyroidism & $33(29.7)$ & $4(3.6)$ & $36(39.1)$ & $3(3.3)$ \\
\hline Proteinuria & $33(29.7)$ & $6(5.4)$ & $25(27.2)$ & $5(5.4)$ \\
\hline Mucositis oral & $28(25.2)$ & $7(6.3)$ & $42(45.7)$ & $8(8.7)$ \\
\hline Hemorrhage & $27(24.3)$ & $4(3.6)$ & $22(23.9)$ & $4(4.3)$ \\
\hline Pharyngalgia & $25(22.5)$ & $1(0.9)$ & $23(25.0)$ & $0(0)$ \\
\hline Vomiting & $16(14.4)$ & $1(0.9)$ & $22(23.9)$ & $3(3.3)$ \\
\hline Weight loss & 15 (13.5) & $0(0)$ & 10 (10.9) & $0(0)$ \\
\hline Nausea & $13(11.7)$ & $1(0.9)$ & $20(21.7)$ & $3(3.3)$ \\
\hline Rash & $11(9.9)$ & $0(0)$ & $47(51.1)$ & $3(3.3)$ \\
\hline Hoarseness & $11(9.9)$ & $0(0)$ & $9(9.7)$ & $1(1.1)$ \\
\hline Dyspnea & $9(8.1)$ & $1(0.9)$ & 11 (11.9) & $0(0)$ \\
\hline Headache & $9(8.1)$ & $0(0)$ & $9(9.7)$ & $0(0)$ \\
\hline Dizziness & $6(5.4)$ & $2(1.8)$ & $6(6.5)$ & $1(1.1)$ \\
\hline Abdominal pain & $6(5.4)$ & $1(0.9)$ & $5(5.4)$ & $2(2.2)$ \\
\hline Constipation & $5(4.5)$ & $1(0.9)$ & $7(7.6)$ & $0(0)$ \\
\hline Conjunctivitis & $3(2.7)$ & $0(0)$ & $12(13.0)$ & $1(1.1)$ \\
\hline Tinnitus & $2(1.8)$ & $0(0)$ & $3(3.3)$ & $0(0)$ \\
\hline Palpitation & $1(0.9)$ & $0(0)$ & $3(3.3)$ & $0(0)$ \\
\hline \multicolumn{5}{|c|}{ Laboratory examination } \\
\hline Pneumonitis & $2(1.8)$ & $0(0)$ & $7(7.6)$ & $3(3.3)$ \\
\hline Hyperbilirubinemia & $10(9.0)$ & $0(0)$ & $20(21.7)$ & $2(2.2)$ \\
\hline AST elevation & $9(8.1)$ & $0(0)$ & 19 (20.7) & $4(4.3)$ \\
\hline Hyponatremia & $8(7.2)$ & $1(0.9)$ & 10 (10.9) & $0(0)$ \\
\hline Creatinine elevation & $7(6.3)$ & $0(0)$ & $8(8.7)$ & $0(0)$ \\
\hline
\end{tabular}

Page $17 / 22$ 


\begin{tabular}{|c|c|c|c|c|}
\hline ALT elevation & $6(5.4)$ & $0(0)$ & $22(23.9)$ & $3(3.3)$ \\
\hline Hypokalemia & $4(3.6)$ & $1(0.9)$ & $8(8.7)$ & $1(1.1)$ \\
\hline Hypoalbuminemia & $4(3.6)$ & $0(0)$ & $4(4.3)$ & $0(0)$ \\
\hline CKMB elevation & $4(3.6)$ & $1(0.9)$ & $6(6.5)$ & $1(1.1)$ \\
\hline Thrombocytopenia & $4(3.6)$ & $0(0)$ & $28(30.4)$ & $4(4.3)$ \\
\hline Anemia & $1(0.9)$ & $0(0)$ & $15(16.3)$ & $2(2.2)$ \\
\hline Neutropenia & $1(0.9)$ & $0(0)$ & $30(32.6)$ & $2(2.2)$ \\
\hline Leukopenia & $1(0.9)$ & $0(0)$ & $35(38.0)$ & $3(3.3)$ \\
\hline \multicolumn{5}{|l|}{ Dose modification } \\
\hline \multirow[t]{2}{*}{ Dose reduction } & 1 level & 2 level & 1 level & 2 leve \\
\hline & $4(3.6)$ & $1(0.9)$ & $5(5.4)$ & $0(0)$ \\
\hline Suspension & $4(3.6)$ & & $6(6.5)$ & \\
\hline
\end{tabular}

Tables 2 to 4 are not available with this version.

Figures 
23 excluded

7 had mixed small cell lung cancer

11 had local treatment for the target lesions

5 had other malignances in previous 5 years

\section{3 patients enrolled}

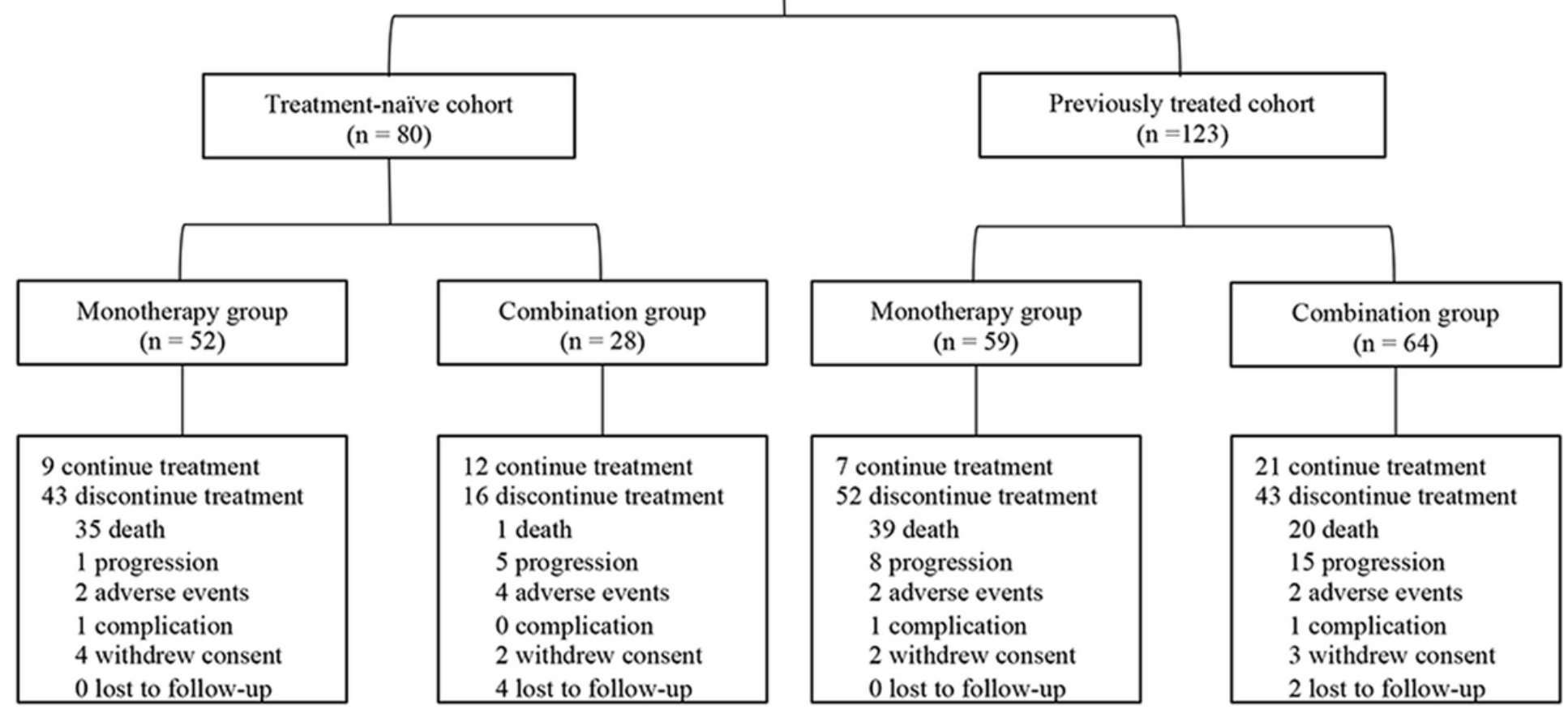

Figure 1

CONSORT diagram of the study population selection for advanced NSCLC.

A

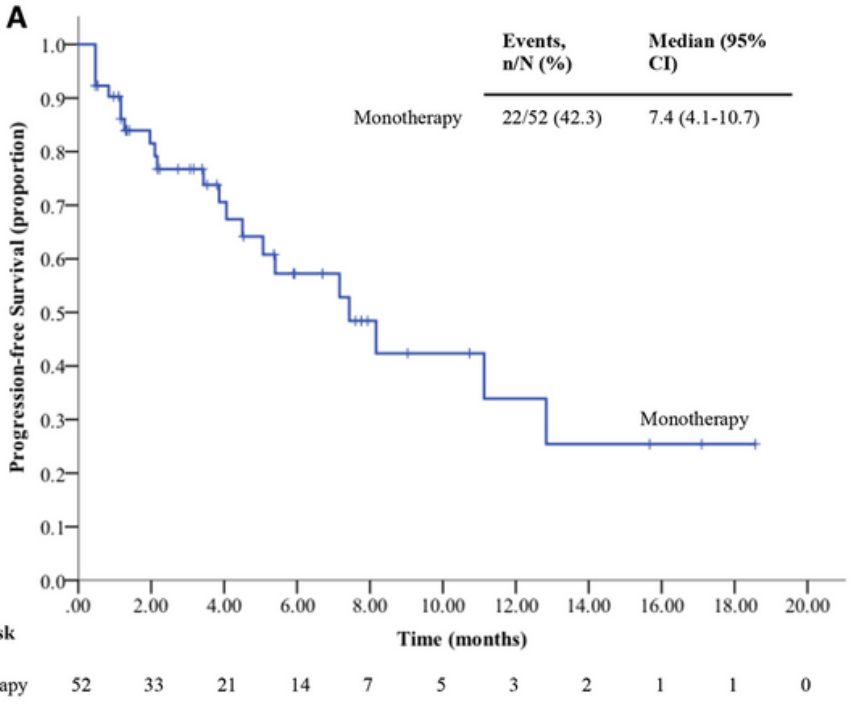

B

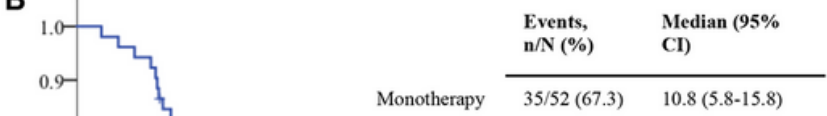

No. at Risk

Monotherapy

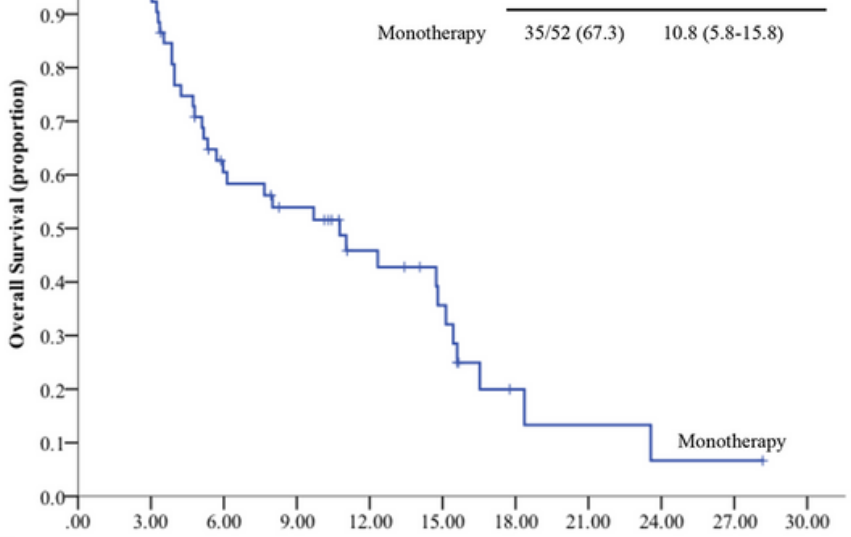

Time (months)

Figure 2 
Kaplan-Meier curve for progression-free survival (Panel A) and overall survival (Panel B) in the treatment-naïve monotherapy population. $\mathrm{Cl}$, confidence interval.

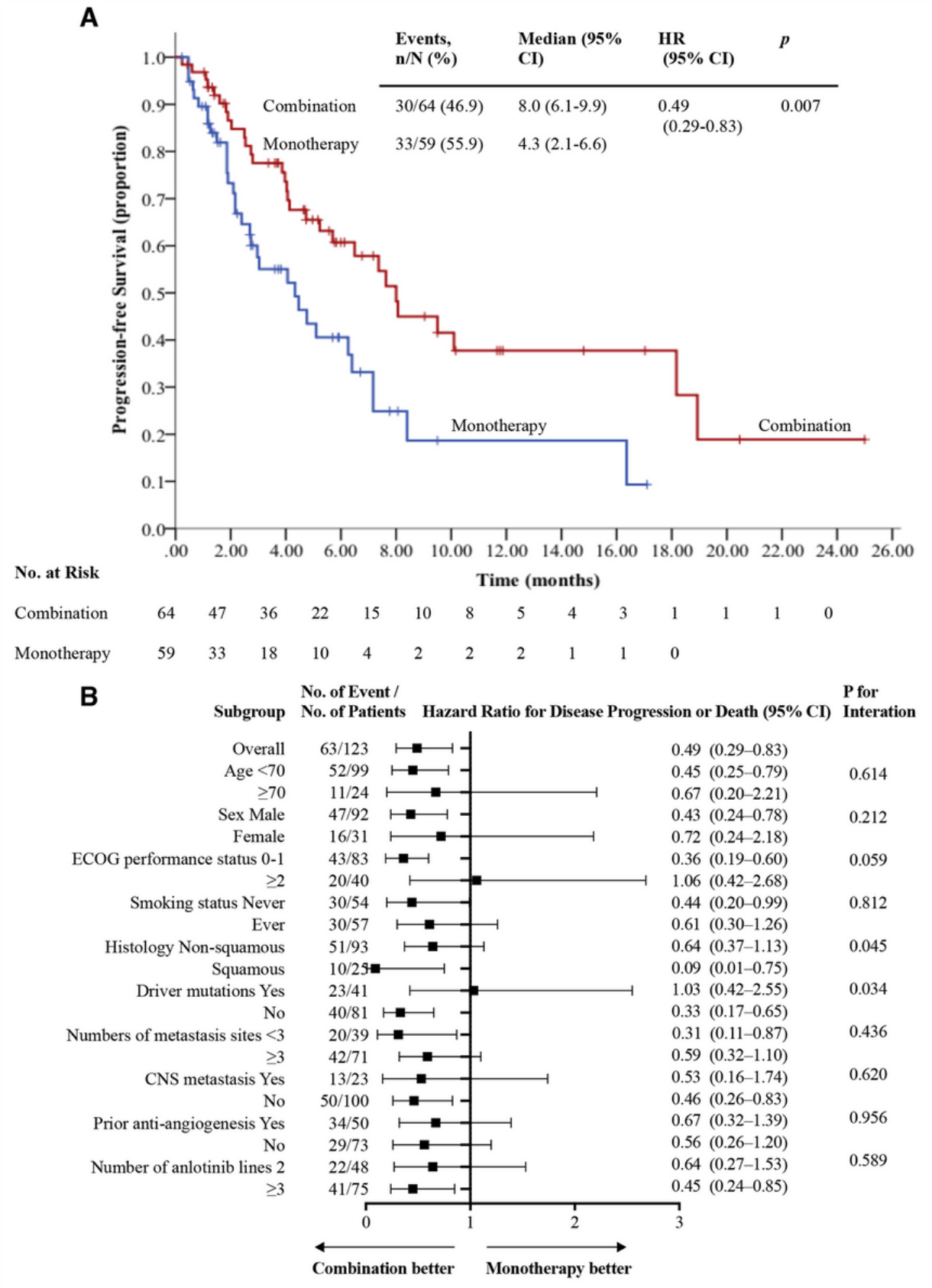

\section{Figure 3}

Kaplan-Meier curve for progression-free survival in the previously treated population (Panel $A$ ) and subgroup analyses of progression-free survival (Panel B). Cl, confidence interval; HR, hazard ratio. 


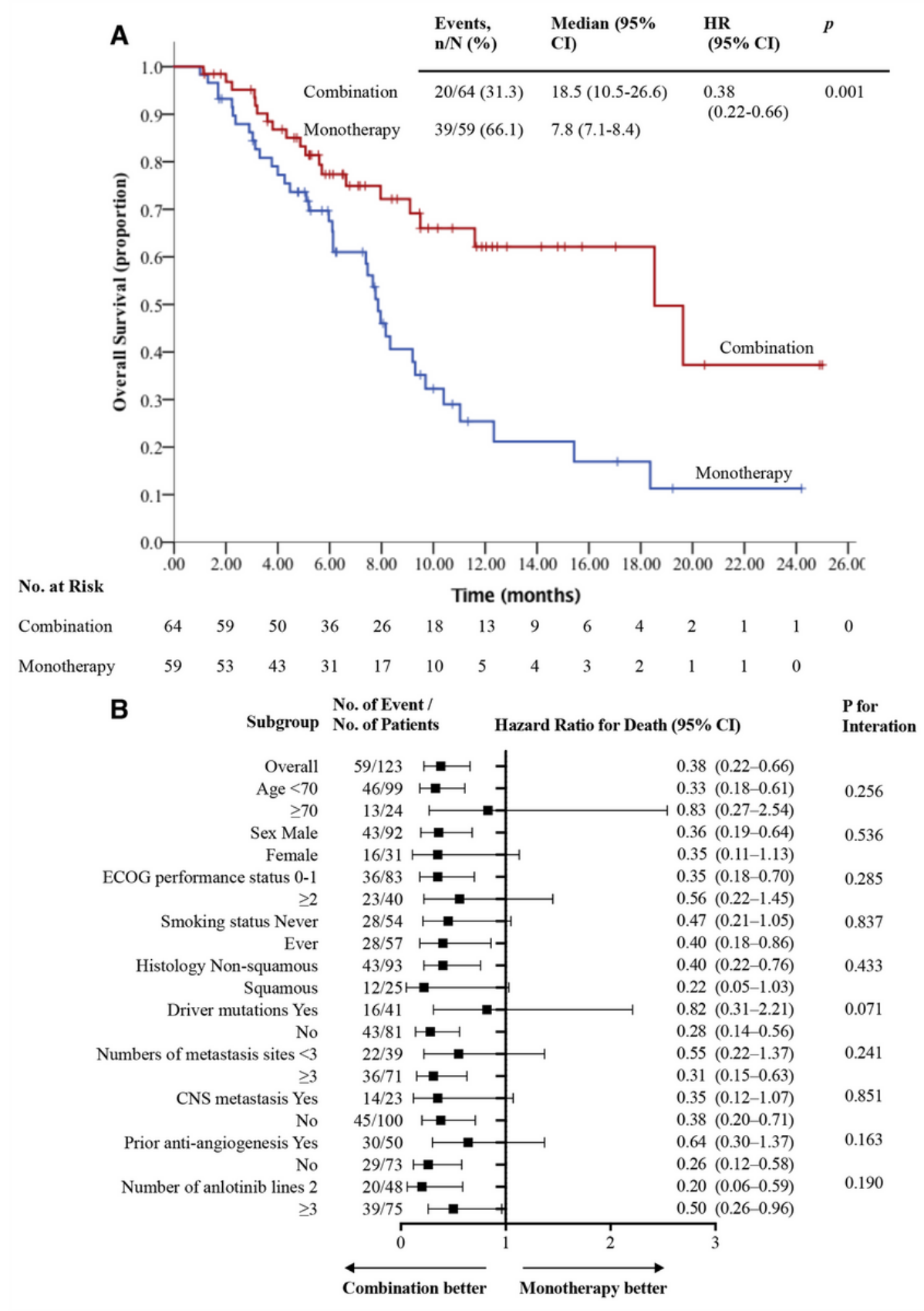

\section{Figure 4}

Kaplan-Meier curve for overall survival in the previously treated population (Panel A) and subgroup analyses of overall survival (Panel B). Cl, confidence interval; HR, hazard ratio. 


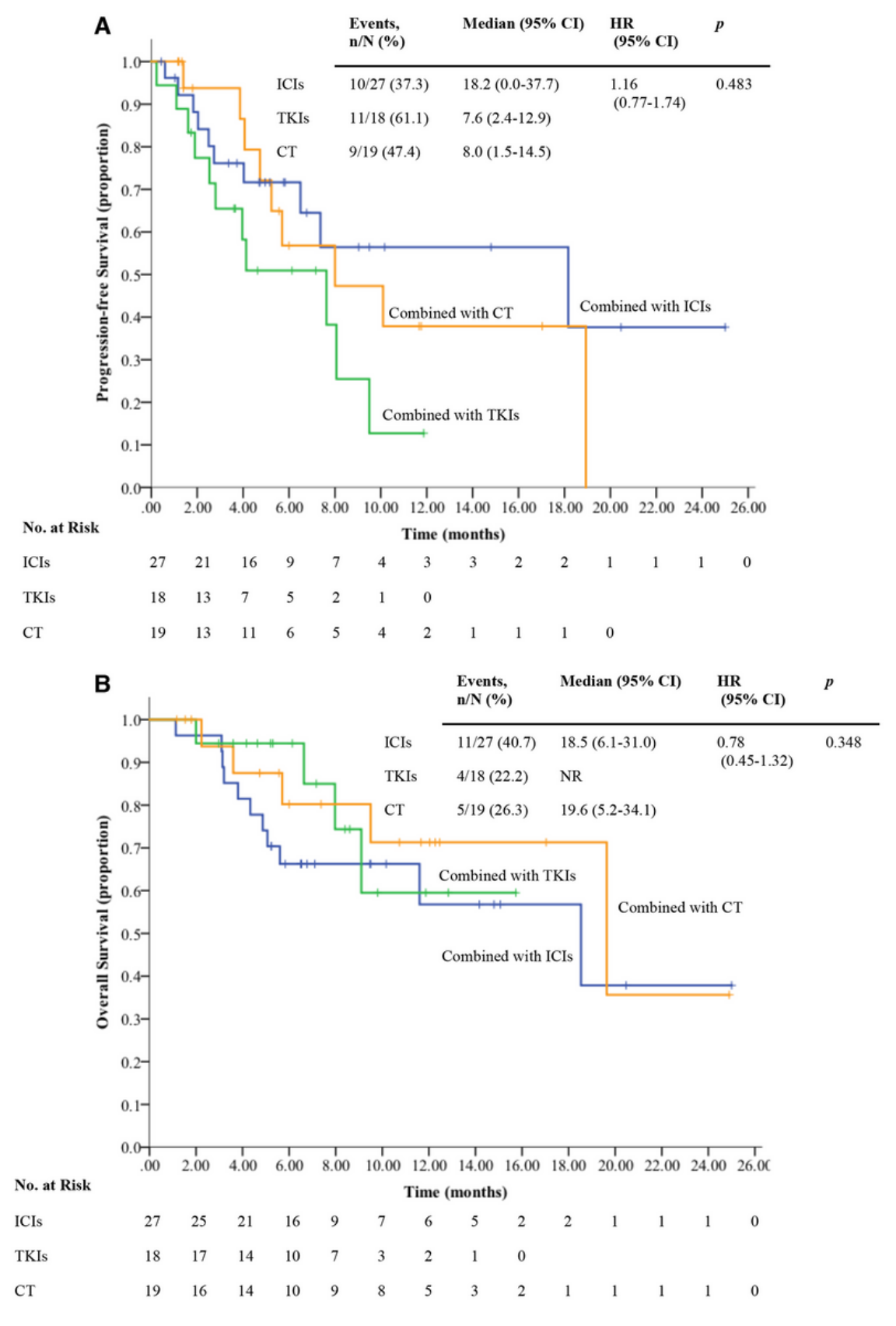

Figure 5

Kaplan-Meier curve for progression-free survival (Panel A) and overall survival (Panel B) in the previously treated combination population. $\mathrm{Cl}$, confidence interval; $\mathrm{HR}$, hazard ratio; ICl, immune-checkpoint inhibitor; TKI, tyrosine kinase inhibitor; CT, chemotherapy.

\section{Supplementary Files}

This is a list of supplementary files associated with this preprint. Click to download.

- SupplementalTable.docx 